\title{
Editorial
}

Trauma Berufskrankh 2019 $21: 1$

https://doi.org/10.1007/s10039-019-0422-2

c) Springer Medizin Verlag $\mathrm{GmbH}$, ein Teil von Springer Nature 2019

Die Bevölkerung altert ... und „verschleißt“: Der endoprothetische Ersatz großer Gelenke spielt daher eine zunehmende Rolle in der Erhaltung schmerzfreier Mobilität und Teilhabe. Statistische Datensätze dokumentieren hohe Wachstumsraten der Endoprothesenimplantationen und spiegeln sowohl Anspruch und Erwartungshaltung der Patienten als auch wachsende medizinische Möglichkeiten wider. Der andauernde Fortschritt in der Evolution von Endoprothesen und Implantationstechniken und der Zusammenschluss der Orthopädie und Unfallchirurgie zu einem Fach eröffnen zunehmend die Frage, ob eine Trümmerfraktur großer Gelenke beim alten Patienten nicht auch primär - und mit Vorteil - mit einer Endoprothesenimplantation versorgt werden könnte.

Die Ergebnisse nach sekundärer endoprothetischer Versorgung bei Osteosyntheseversagen sind allerdings häufig auch zufriedenstellend und die Komplikationsraten bei Primärimplantation in Abhängigkeit von der Lokalisation hoch. Jede Revisionsoperation stellt zudem für diese häufig multimorbiden Patienten eine erneute - und im Extremfall vital bedrohliche - Belastung dar. Der Funktionsanspruch dieser Patienten ist im Sinne einer schmerzfreien Mobilisierbarkeit unter Vollbelastung eines stabilen Gelenks nicht selten limitiert. Systematische Datenlagen hierzu sind noch spärlich. Die Qualitätssicherung mit Routine- und Registerdaten, gemeinsame Longitudinalbeobachtungen und Analysen großer Patientenpopulationen durch wissenschaftliche Fachgesellschaften und Kostenträger sowie internetbasierte PROMS („Patient Related Outcome Measurements") durch Patienten oder Angehörige werden hier

\author{
R. Hoffmann · M. Kremer \\ BG Unfallklinik Frankfurt am Main gGmbH, Frankfurt/M., Deutschland
}

\section{Endoprothetik nach Trauma}

zukünftig einen wertvollen Beitrag leisten.

Im Bereich der berufsgenossenschaftlichen Heilverfahren spielen besonders posttraumatische Zustände beim Gelenkersatz eine Rolle. Hier verkomplizieren Achsfehlstellungen, Gelenkkontrakturen oder -instabilitäten, Knochendefekte und instabile Weichteilsituationen die Ausgangsbedingungen. Jeder dieser Punkte erhöht bereits für sich genommen das Risiko für eine erfolgreiche Endoprothesenimplantation. Meist treten sie mehr oder weniger in Kombinationen auf. Die Endoprothesenimplantation bei posttraumatischen Zuständen zeigt daher auch in der Hand des Erfahrenen nicht immer vorhersehbar gute oder reproduzierbare Ergebnisse. Jeder Fall ist anders und bedarf differenzierter Konzepte und Lösungen. Die Ausgangsbedingungen sind regelhaft schlechter als bei einem „regulären“ Endoprothesenwechsel. Entsprechend anspruchsvoll sind individuelle Fallanalysen, Planungen und Umsetzungen. Die Erwartungshaltung der Patienten muss angepasst werden. Der erforderliche Aufwand ist personell und implantattechnisch erheblich und zeit- wie kostenintensiv. Eine Konzentration auf spezialisierte Zentren erscheint daher naheliegend und sinnvoll. Hier ist in erster Linie an diejenigen EPZmax zu denken, die einen ausgewiesen hohen Anteil an Revisionsendoprothetik durchführen und - sofern es sich um Arbeits- oder Wegeunfallfolgen handelt - mit den bg-lichen Heilverfahren vertraut sind.

Das vorliegende Schwerpunktheft gibt einen Überblick über den aktuellen und etablierten Stand der Endoprothetik nach Trauma und stellt Innovationen der Exoprothetik der oberen Extremität dar.
Ihre

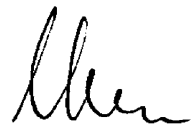

Prof. Dr. Dr. Reinhard Hoffmann

Ärztlicher Direktor

BG Unfallklinik Frankfurt am Main gGmbH

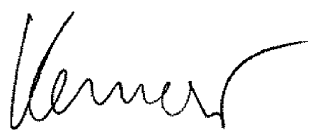

Dr. med. Michael Kremer

Sektionsleiter Endoprothetik/EPZmax

\section{Korrespondenzadresse}

Prof. Dr. Dr. R. Hoffmann

BG Unfallklinik Frankfurt am Main gGmbH Friedberger Landstr. 430, 60389 Frankfurt/M., Deutschland

Reinhard.Hoffmann@bgu-frankfurt.de

Interessenkonflikt. R. Hoffmann und M. Kremer geben an, dass kein Interessenkonflikt besteht. 